Article

\title{
System Integrity Protection Scheme (SIPS) Development and an Optimal Bus-Splitting Scheme Supported by Phasor Measurement Units (PMUs)
}

\author{
Zoran Zbunjak $^{1, *(1)}$ and Igor Kuzle ${ }^{2}$ (1) \\ 1 Croatian Transmission System Operator Ltd., Matulji 51211, Croatia \\ 2 Department of Energy and Power Systems, Faculty of Electrical Engineering and Computing, \\ University of Zagreb, Zagreb 10000, Croatia \\ * Correspondence: zoran.zbunjak@hops.hr; Tel.: +385-51-710-733
}

Received: 14 July 2019; Accepted: 30 August 2019; Published: 3 September 2019

check for updates

\begin{abstract}
System integrity protection schemes (SIPS) are schemes that can, under potentially hazardous conditions, prevent a complete blackout of endangered parts of an electrical power system (EPS). The main objective of SIPS is to monitor the state of the power transmission network in real time and to react in emergency cases. This paper explores the use of phasor measurement unit (PMU) technology for the development of SIPS as a part of wide-area monitoring, protection, and control (WAMPAC) systems. A new SIPS development method is described using the experience from the real-time operation. The developed optimal bus-splitting scheme identifies potential actions that can eliminate or reduce power system overloads and protect the integrity of the power system. An optimal bus-splitting scheme based on a DC power flow model and PMU measurements is given as an example and is explained and tested on an IEEE 14 bus test system. Conducted simulations indicate that the described SIPS methodology supported by the PMU measurements can mitigate potential overloads of the observed network part.
\end{abstract}

Keywords: optimal bus-splitting scheme; phasor measurement unit; power system analysis; smart transmission grid; system integrity protection schemes; wide-area monitoring; protection and control

\section{Introduction}

System integrity protection schemes (SIPS) are schemes that can, under potentially hazardous conditions, prevent a complete blackout of endangered parts of an electrical power system (EPS). Paper [1] highlights the need for providing research on impacts of renewables on EPSs and the corresponding protection and control strategies in order to mitigate the various negative effects. These requirements are trying to be reached with the development of smart transmission grid (STGs). An STG implies performance of automated processes based on measurement, control, protection, and telecommunication systems supported by smart technologies in order to maintain a secure power network state. According to [2] one of the solutions for large-scale renewable integrations is to increase the flexibility of the EPS by using SIPS. Advanced control and SIPS are specific areas where significant improvements can be achieved using PMU technology according to [3]. The mentioned technology was developed back in the 1980s [4,5] and has been implemented in different aspect of EPS operation, including an extension of the local relay protection functions and some advanced system protection functions (e.g., out of step [6], all frequencies protection [7], and reduction of the excessive standing phase angle difference during the system restoration process [8]).

Development of smart technologies has placed increasing demand on the speed of data exchange and processing [9]. The biggest advantage of PMU technology is the capability to transfer large 
amounts of data that are synchronized with GPS timestamps from different parts of the EPS in real time. The undertaken research within the presented paper refers to several related problems identified in the literature:

- Congestion in parts of the power transmission network due to the electricity market conditions in its control [10],

- High shares of electricity production from renewable sources, which is characterized by variable generation and difficult scheduling [11],

- Changes in the nature and structure of electricity consumption (electrical vehicles, etc.), and

- Coordination of local relay protection systems in different parts of the EPS [12].

Solutions to the specified research problems are being looked at with the application of PMU technology in the form of developing SIPS [13]. The main contribution of this work is merging SIPS with the additional capabilities that PMU measurements can bring. PMU placement is a well-researched and developed area, as it appeared in the initial use of PMU technology [14,15]. Paper [16] introduces complete and incomplete system observability placement techniques. Incomplete observability is described with the concept of unobservability depth that directly affects the total number of required PMUs. Paper [17] introduces a critical location method together with the observability method. The critical locations method consists of determining the buses with a large number of elements or with limited voltage values that may affect the security of the system. These papers conclude that PMU technology application in SIPS requires PMU placement that allows complete system observability.

Large modern EPSs have become vulnerable to overload problems due to their interconnectedness. The impact of line disconnections in one part of the network must sometimes be observed in several neighbouring power systems. Accurate, but perhaps unnecessary, operation of local overload protection in one part of the network can lead to significant consequences for the entire interconnection. Overload protection is typically adjusted conservatively to preserve the asset it protects. Such conservative parameters have their reasons, but they usually do not take into account the importance of overload in relation to the security of the whole system. Without use of SIPS that will protect a larger part of the EPS, and thus improve the coordination of local overload protections, it is not possible to eliminate potential large blackouts.

This paper presents the results and experience from the development process of a SIPS method for a real operation problem utilizing the availability of full PMU coverage of the observed transmission system. The research hypothesis is defined as follows: development and use of SIPS, supported by PMU technology, can maintain the integrity of a larger part of an electric power system, improve coordination of local relay protection systems, and mitigate potential congestions in the power network.

Optimal bus-splitting schemes together with overload and congestion mitigation schemes are studied within this paper. This paper describes contributions in the field of congestion management and enhancement of security with the usage of SIPS supported by PMU measurements. It is structured as follows: Section 1 provides the introduction, followed by Section 2, which describes SIPS in general. Section 3 describes the developed method, while in Section 4, a mathematical description of an optimal bus-splitting problem is given. Next is Section 5 where an IEEE 14 bus case study is described, and the conclusions are finally drawn in Section 6.

\section{System Integrity Protection Scheme (SIPS)}

In 1996 a report on special protection schemes was published [18]. This report included more than 100 protection schemes used around the world. In 2010 a report on SIPS was published [19]. According to the report, SIPS deals with congestion, thermal overload, and voltage, frequency, and angular instability problems. These stated problems can be mitigated using different types of protection schemes.

The use of transmission switching as a corrective action for resolving congestion was first mentioned in 1980 [20,21]. An overview of works dealing with transmission-switching analyses from 1980 to 1999 are presented in [22]. Line and transformer switching, together with bus-splitting 
actions, are described in the given literature as models for network topology changes. The developed optimal transmission switching protection scheme in this paper uses only bus-splitting mechanisms, whose search for a solution is far more mathematically demanding than the search for individual, disconnected elements, as stated in [23].

Special protection schemes are defined in [18] as schemes designed to identify specific events that can cause unusual problems in the operation of the EPS and to undertake advanced, predefined measures that will neutralize the detected events. SIPS, supported by PMU technology and applied to a wide area, are developed in this paper, and a general description is depicted in Figure 1.

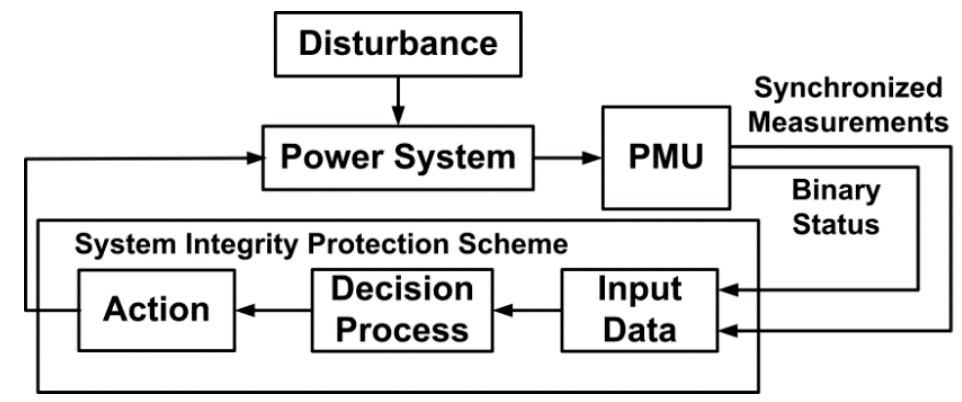

Figure 1. System integrity protection scheme (SIPS) based on phasor measurement unit (PMU) synchronized measurements.

\section{System Integrity Protection Scheme Development Method}

SIPS are designed based on power system analysis by defining requirements that they must meet. They are made based on a power system's response to the recorded events or congestions, security assessment, heuristic methods, and AC power flow analysis. A flowchart of the proposed SIPS development method is shown in Figure 2. The flowchart is described in the following Sections 3.1-3.6.

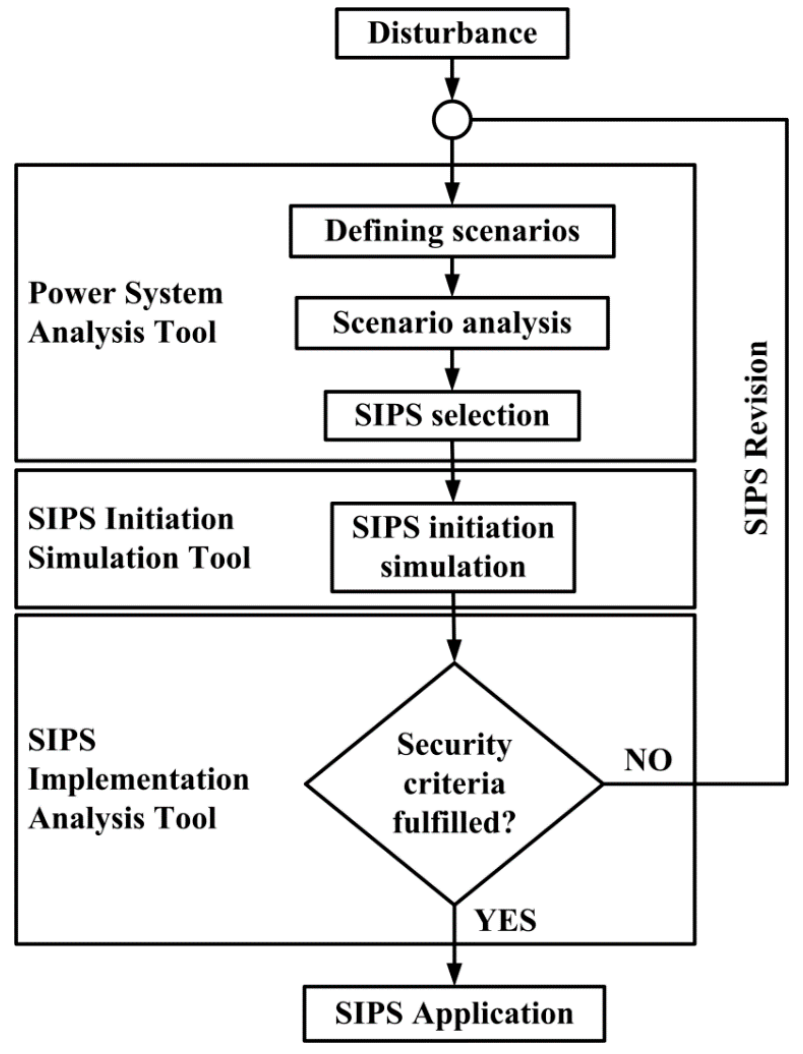

Figure 2. Flowchart of the created method. 


\subsection{Disturbance}

Unpredictable disturbances or events in the network indicate the need for a detailed analysis of the power system. For such disturbances or events, it is required to record all input parameters and system responses that can be analysed using the specialized tools for power system analysis. Large disturbances or events in the power network can surely serve as idea originators for the making of SIPS.

\subsection{Scenario Definition for Power System Analysis}

The first step in SIPS development is to define a number of scenarios that include representative disturbances in the observed part of the system. There are different principles for determining scenarios in the analysis of power networks. Principles can be applied to seasonal (summer/winter), hydrological (good/bad hydrology), or temporal criteria (night/day) depending on many different network factors: the location of the generating units, topology and scheduling of the transmission network, load distribution, and so on. Selection criteria for defining the analysis scenarios depend on experience and expert knowledge of the observed part of the power system.

\subsection{Scenario Analysis}

The second step in SIPS development relates to a detailed analysis of the power system. Performing an AC power flow analysis is suggested because current applications of the described method are designed to perform offline analyses that are not time critical, unlike some faster analysis that can be used in real-time applications like DC power flow analyses [24]. It is recommended to analyse the basic conditions of each individual scenario defined in the previous step. These analyses will indicate the initial potential risks of the observed part of the network. It is necessary to analyse in detail each potential initial risk (i.e., high network load or voltage and angular deviations).

After analysing the basic conditions of each scenario and defining the initial potential risks, in terms of high loading or voltage and angular deviations of the observed parts of the network, $\mathrm{N}-1$ analyses can be accessed. Contingency analyses (N-1) can be extended to multiple element outages if necessary in the form of $\mathrm{N}-\mathrm{k}$ analyses, where $\mathrm{k}$ denotes the number of excluded elements.

It is recommended to perform N-1 analyses of each scenario for all elements in proximity to the endangered elements, defined as the initial potential risks. In the analysing process it is necessary to record all the N-1 or N-k analyses that have caused significant load increases of individual network elements.

\subsection{SIPS Selection}

During selection of any kind of protection scheme it is necessary to have expert knowledge of the existing local relay protection system in order not to unnecessarily disturb its coordination by implementing new protection schemes. Mutual local relay protection and SIPS coordination will lead to successful mitigation and prevention of possible disturbances over a wide area and will maintain the security and integrity of the system.

SIPS proposed in this paper are focused on corrective bus-splitting schemes that maintain the existing level of power system generation and load. SIPS that do not maintain the existing level of power system generation and load, like load shedding and generation reduction schemes, will be studied in future works where there is no possibility of using bus-splitting schemes.

Bus-splitting schemes are characterized by retaining all elements connected to the power network. They use the principle of load redistribution in order to mitigate the load in the endangered parts of the network. In case of their usage there should be no consequences for participants connected to the network.

Mitigating congestion by changing the network topology essentially refers to the separation or integration of the EPS in differently connected network parts. System separation or integration is performed by separating or integrating different bus systems or their sections in parts where their primary and secondary equipment design allows it. It is important to emphasize that any separation 
or integration of the system will impact the power flow and voltage conditions in the observed part of the network. In case of implementing any considered measure, it is important to carry out detailed system security analyses.

While developing bus-splitting schemes, it is possible that none of the analyses indicates the possibility of mitigating any bus-splitting actions. This can happen for various reasons if the primary system is not built flexibly enough or the secondary systems cannot adequately respond to the requirements of the developed model. In these cases, it is recommended to seek mitigation solutions in other SIPS, even those that do not maintain the existing load and level of power system generation.

While defining SIPS it is necessary to assign each scheme a unique label. This description implies defining substations and highlighting the type of SIPS applied. If the bus-splitting scheme is chosen, it is necessary to exactly define which element needs to be connected to which bus. A template for defining the bus-splitting scheme is given in Table 1.

Table 1. Template for defining bus-splitting scheme actions.

\begin{tabular}{cc}
\hline SIPS & SIPS Label \\
\hline Substation & Substation name \\
\hline Bus I & List of bays that need to be connected to bus I \\
Bus II & List of bays that need to be connected to bus II \\
$\ldots$ & \\
Bus N & List of bays that need to be connected to bus N \\
\hline
\end{tabular}

\subsection{SIPS Initiation Conditions}

Defining SIPS initiation conditions consists of defining two sets of conditions that will uniquely determine the initiation of necessary actions. The first group of conditions refers to the comparison of actual analogue measured values obtained using synchronized phasor measurement units with predefined values that are specified based on a detailed analysis. Another group of conditions refers to the comparison of the required connected or disconnected status of individual elements in a network with predefined ones also based on detailed analyses. Both sets of conditions consist of their elements for starting or blocking SIPS. The launching principle of a single scheme lies in the fact that all conditions must be satisfied in order to run it.

The SIPS simulation can be initiated and accessed after defining the preliminary activities and design requirements. The Matlab [25] environment was used as a simulation tool. After the simulation phase it is possible to revise the designed SIPS with the defined requirements if certain irregularities in their functioning are noticed during the simulation step. Figure 3 shows a block scheme for initiating the SIPS simulation in Matlab.

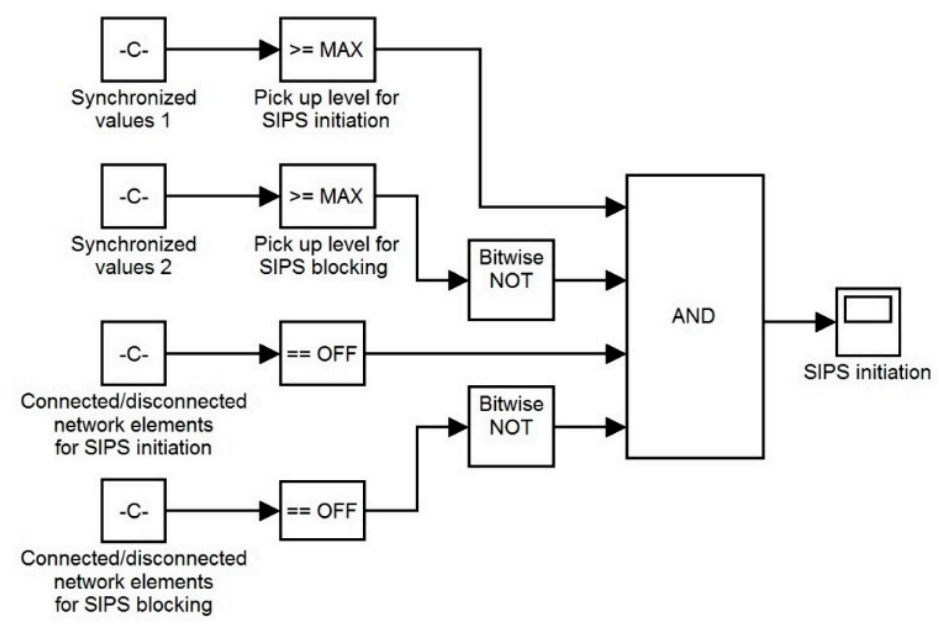

Figure 3. Block scheme of the developed Matlab simulation model. 
After developing SIPS and describing the bus-splitting scheme, an algorithm for SIPS initiation is developed. The algorithm has a high reliability and start-up accuracy, based on a double check of the start-up conditions separated by time delay execution. After verifying the switch state of the network elements and double checking the start-up conditions, the algorithm launches the selected SIPS based on defined activities. Upon execution, the algorithm continues with its work by checking the termination of the start-up conditions. Afterwards, unblocking of the local relay protection backup stages is issued, the data sampling time is moved to the next data sample according to the defined time step, and the algorithm returns to the beginning of the loop where it re-checks the switch state of the network elements and verifies the collected measured value. A flow diagram of the developed algorithm for SIPS initiation is presented in Figure 4.

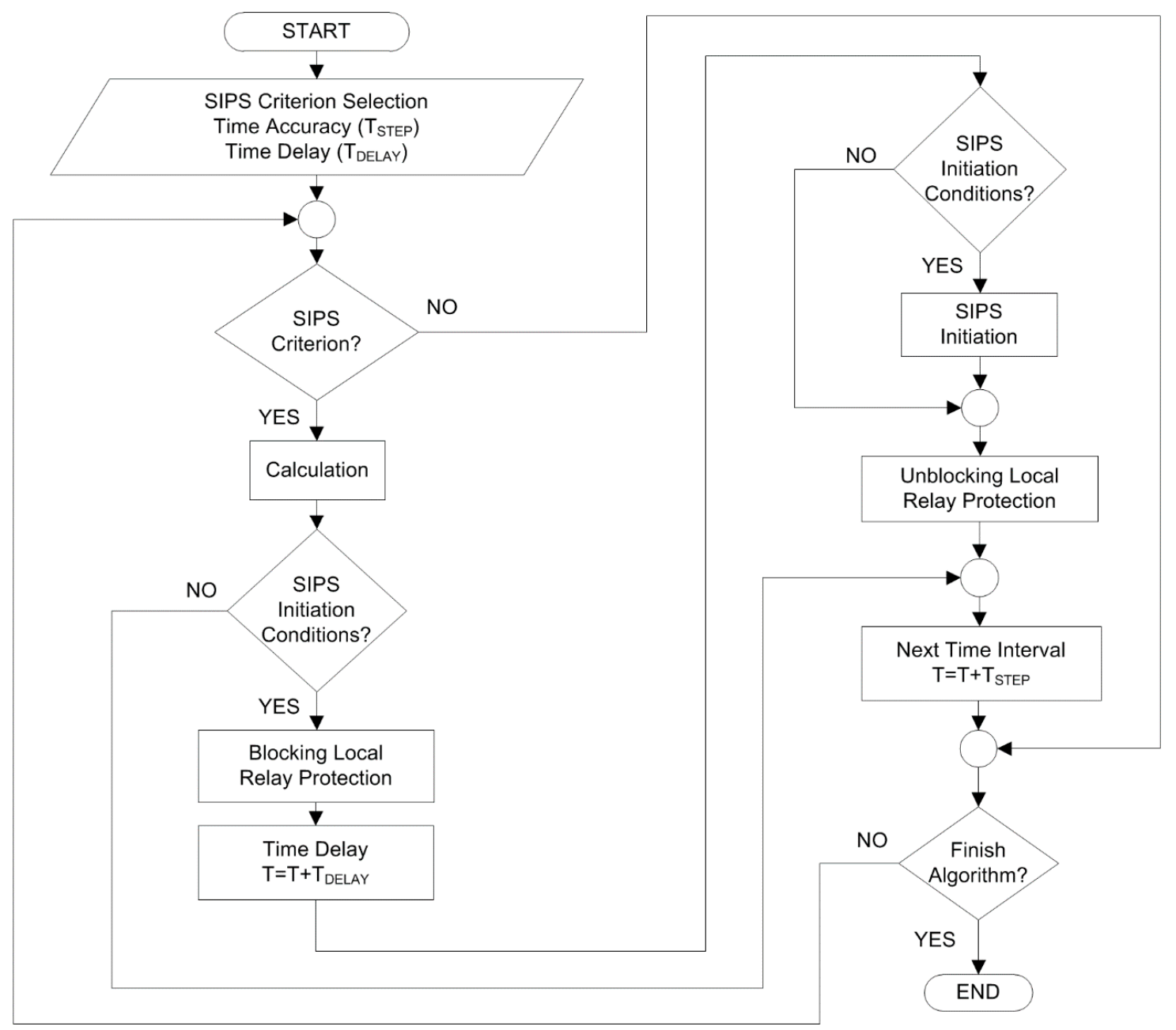

Figure 4. Flow diagram of the developed algorithm for SIPS initiation.

\subsection{Power Network Security Assessment with and without SIPS Implementation}

Each SIPS application influences the power flows and the voltage conditions in the observed part of the transmission network. For this reason, it is important to carry out a detailed analysis of the entire system after the implementation of SIPS. Analyses presented in this paper include comparisons of voltage conditions and power flows paired together with the generation, consumption, and levels of loss for the system, with and without the use of the designed SIPS. Each of the mentioned comparisons is presented as a graphical chart, from which it is possible to extract the advantages and disadvantages of any particular scheme. Choosing the final scheme is based on set criteria that can best fit the needs, for example, a scheme that causes the least losses in the observed network part without endangering any segment of the network or a scheme that allows for maximum evacuation of the produced wind energy. 


\section{Mathematical Description of the Optimal Bus-Splitting Problem}

The developed algorithm uses mixed integer linear programming (MILP) based on branching and bounding methods when searching for an optimal solution. MILP explores optimization problems within the given constraints of the observed system. Optimization can be based on finding the maximum or minimum of the set objective function. The decision-making process is made based on the given constraints in the form of equations or inequalities. In MILP, decisions can take values from a set of real or integer numbers. The method of branching and bounding is based on the principle of successfully resolving the objective function until the decision values reach the optimal solution. The mathematical model of the used algorithm is based on the DC power flow calculation, which has three assumptions:

- line resistance $R_{L}$ is negligible in relation to line reactance $X_{L}$,

$$
R_{L} \ll X_{L}
$$

- $\quad$ per unit voltage $\left|V_{N}\right|$ is the same for all nodes,

$$
\left|V_{N}\right|=1 \text { p.u.; }
$$

- $\quad$ voltage angles difference $\delta_{i}-\delta_{j}$ of the adjacent nodes is small, which derives:

$$
\sin \left(\delta_{i}-\delta_{j}\right) \approx\left(\delta_{i}-\delta_{j}\right) ; \cos \left(\delta_{i}-\delta_{j}\right) \approx 1 .
$$

Taking into account these assumptions will affect the accuracy of the DC model. According to [26], the rough total error of the DC model is $5 \%$ compared to the more accurate nonlinear AC model. Though the accuracy of the DC model is smaller than that of the AC model, it is characterized by the simplicity and speed of the calculation conditioned by its linearity, which is why it is proposed in making SIPS.

Unlike the load-shedding or generation reduction schemes, the goal of transmission-switching protection schemes is to maintain the existing level of production and consumption in the observed power system. An optimal bus-splitting protection scheme based on a linear DC power flow model and synchronized phasor measurements is given below. The main contribution of developed algorithm is the introduction of an adjustment factor $k_{P e}$ in order to replace the inequality of the DC network model with respect to the actual state of the power system. The adjustment factor is calculated in real time using synchronized phasor measurements. The model features exceptional performance speeds that enable its use in SIPS and, consequently, preserves the security of the power system in its entirety.

Note the example of a substation with six initially defined elements in Figure 5. The substation consists of one generator, one load, one transformer, and three lines. All of the above elements are connected to one bus.

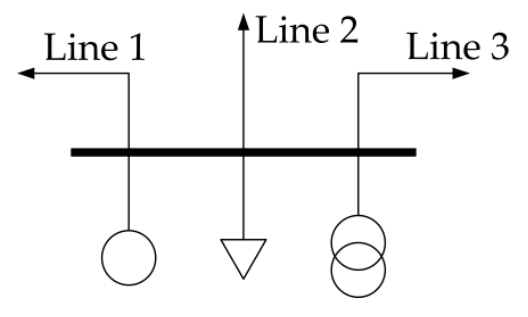

Figure 5. An example of a bus with six initially defined elements.

In order to mathematically describe the optimal bus-splitting problem it is necessary to create an equivalent model for each substation. The equivalent substation model consists of $N$ newly created buses, where $N$ is the total number of initially defined elements associated with the observed substation. 
The total number of initially defined elements represents the total number of all connected generators, loads, transformers, and lines to the observed substation. After creating $N$ new buses, it is necessary to create $E$ new elements, with extremely low impedance, which connect new buses in all possible combinations. New elements, with extremely low impedance, can be called zero-impedance elements. Each new element, connecting the two new buses, is assigned with a decision variable that represents its closed or open state. The total number of new zero-impedance elements per substation is defined by the following expression:

$$
E=\frac{N(N-1)}{2}
$$

For the given example of a substation with six initially defined elements, according to Figure 5, the total number of new zero-impedance elements is fifteen, as shown graphically in Figure 6.

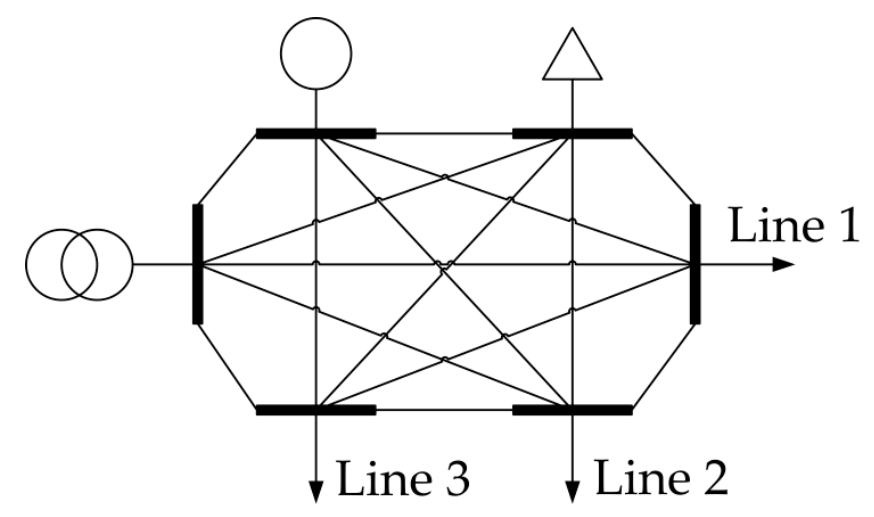

Figure 6. Equivalent bus model with six initially defined elements.

Calculation of the DC power flow model for the described equivalent system can be described by the following nonlinear expression:

$$
P_{e}=\left(\frac{\delta_{i}-\delta_{j}}{X_{e}}\right) \times V A R E_{e} ; e \in E ; i, j \in N ; \delta_{R E F}=0,
$$

where:

- $\quad e$-element tag,

- $\quad i, j$-node tag,

- $P_{e}$-active power flow for element $e$,

- $\delta_{i}, \delta_{j}$-voltage phase angle for node $i$ and $j$,

- $X_{e}$ - element $e$ reactance,

- $V A R E_{e}$ - decision variable which represents element $e$ in the closed or open state, defined as binary value 0 for open or 1 for closed state,

- E-number of elements,

- $\quad N$-number of nodes, and

- $\delta_{R E F}$-reference voltage phase angle.

Expression (5) needs to be linearized according to the terms below in order to the solve optimization problem with MILP methods:

$$
\begin{aligned}
& P_{e} \geq\left(\frac{\delta_{i}-\delta_{j}}{X_{e}}\right)-\left(1-V A R E_{e}\right) \times M_{e} ; e \in E ; i, j \in N ; \delta_{R E F}=0, \\
& P_{e} \leq\left(\frac{\delta_{i}-\delta_{j}}{X_{e}}\right)+\left(1-V A R E_{e}\right) \times M_{e} ; e \in E ; i, j \in N ; \delta_{R E F}=0,
\end{aligned}
$$


where:

- $\quad M_{e}$-linearization factor for element $e$, defined by Expression (8).

Linearization is achieved by introducing value $M$ defined by the following expression:

$$
M_{e}=\left(\frac{\delta_{M A X}-\delta_{M I N}}{X_{e}}\right) ; e \in E,
$$

where:

- $\delta_{M A X}-$ maximum voltage phase angle, and

- $\delta_{M I N}-$ minimum voltage phase angle.

If the decision variable, which represents element $e$ in the closed or open state, is equal to one, then Expressions (6) and (7) result in

$$
P_{e}=\left(\frac{\delta_{i}-\delta_{j}}{X_{e}}\right) ; e \in E ; i, j \in N ; \delta_{R E F}=0,
$$

which corresponds to the definition of the DC power flow calculation when element $e$ is closed. If the decision variable, which represents element $e$ in the closed or open state, is equal to zero, then the active power of the observed element is also equal to zero, and the difference of voltage angles in nodes $i$ and $j$ is limited by the calculated $M_{e}$ value.

The node sum is defined by the following expression:

$$
G_{i}-L_{i}=\sum_{e \epsilon E} P_{e} ; i \in N
$$

where:

- $G_{i}$-active generation power at node $i$, and

- $\quad L_{i}$-active load power at node $i$.

The active power flow constraint of element $e$ with respect to its maximum permissible apparent power is defined by the following expression:

$$
\left|P_{e}\right| \leq k_{P e} \times S_{M A X e} \times V A R E_{e} ; e \in E,
$$

where:

- $\quad k_{P e}$-adjustment factor that is calculated in real time during the overload state, defined by Expression (12),

- $S_{M A X e}$-maximum permissible apparent power of element $e$.

Adjustment factor $k_{P e}$ is introduced in order to compensate for the inaccuracy of the DC power flow model. It is calculated in real time using synchronized phasor measurements

$$
k_{P e}=1-k_{s} \frac{\left|P_{R E A L e}-P_{D C e}\right|}{S_{M A X e}} ; e \in E,
$$

where:

- $\quad k_{s}$-safety coefficient with selected value 1.2,

- $\quad P_{R E A L e}$-real-time measured active power of element $e$ during the overload period using the PMU device, and

- $\quad P_{D C e}$-calculated active power flow of element $e$ based on the DC model during the overload period. 
In order to ensure an optimal and safe bus-splitting solution, it is necessary to set constraints that do not allow initially modelled lines or transformers to be disconnected. Constraints that ensure the connected state of at least two elements on all modelled buses are also mandatory in addition to the above restriction.

The objective function of the described optimization algorithm is to minimize total production according to the following expression:

$$
\operatorname{Min}\left(\sum_{i \in N} G_{i}\right) .
$$

The described optimization algorithm finds the optimal bus-splitting combination by taking into consideration the maximum permissible power flow limits on the network elements. Fulfilment of the assigned constraints has the effect of mitigating initial element overloading. Figure 7 shows a symbolic example of a bus-splitting combination for a bus with six initially defined elements. The equivalent bus model is split on three bus systems, each linking two elements.

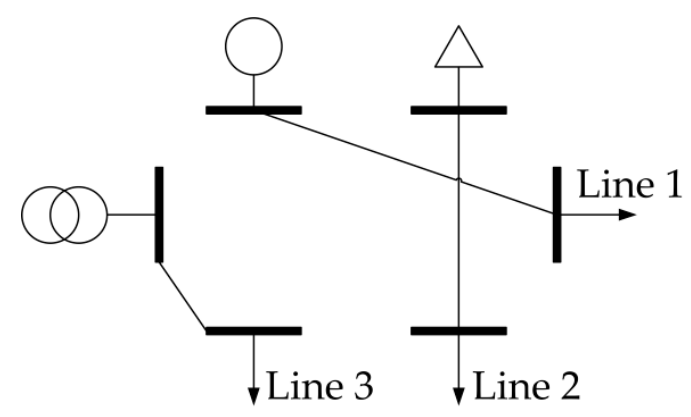

Figure 7. Symbolic example of a bus-splitting combination on three bus systems for a bus with six initially defined elements.

\section{Case Study on an IEEE 14 Bus Test System}

The developed optimal bus-splitting protection scheme based on DC power flow and PMU measurements was tested on an IEEE 14 bus test system, which is shown in Figure 8. The default test system did not have line and transformer power flow limits. Therefore, certain modifications were made. Line and transformer power flow limits were assumed according to Table 2.

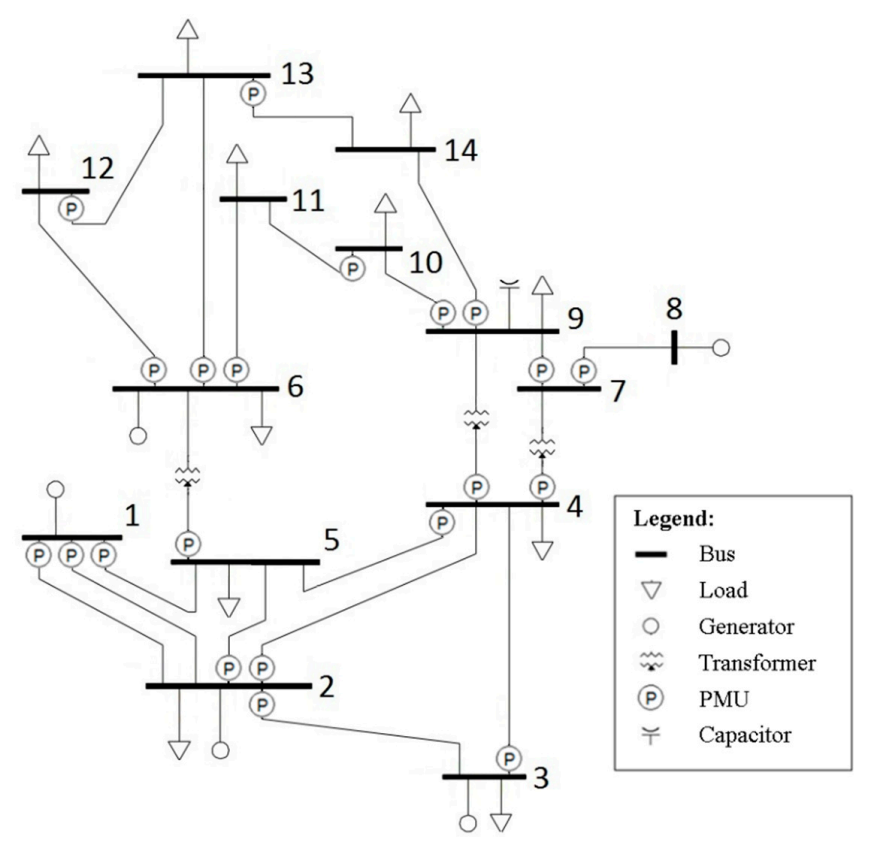

Figure 8. IEEE 14 bus test system with PMU placement for an optimal bus-splitting protection scheme. 
Table 2. Assumed line and transformer power flow limits.

\begin{tabular}{cc}
\hline Elements & Power Flow Limit (MVA) \\
\hline Lines & 130 \\
$1-2(1), 1-2(2), 1-5$, & \\
$2-3,2-4,2-5,3-4,4-5$ & 85 \\
\hline Transformers & \\
$4-7,4-9,5-6$ & 70 \\
Lines & \\
$6-11,6-12,6-13,7-8,7-9$, & \\
$9-10,9-14,10-11,12-13,13-14$ & \\
\hline
\end{tabular}

\subsection{Base Scenario}

The analysis was performed on the base scenario without any changes to the defined test system's topology and consumption. Data for the IEEE 14 bus system were taken from the University of Washington's test case archive [27]. The base scenario was defined with the majority of the generation located on bus 1 and a smaller generation amount in bus 2. Buses 3, 6, and 8 were modelled with synchronous compensators that maintained the network's voltage security. Figure 9 shows a power flow calculation of the base scenario, representing the active power flow and percentage values on lines and transformers. Power flow calculations were performed using PSS®E [28] analysis software. 


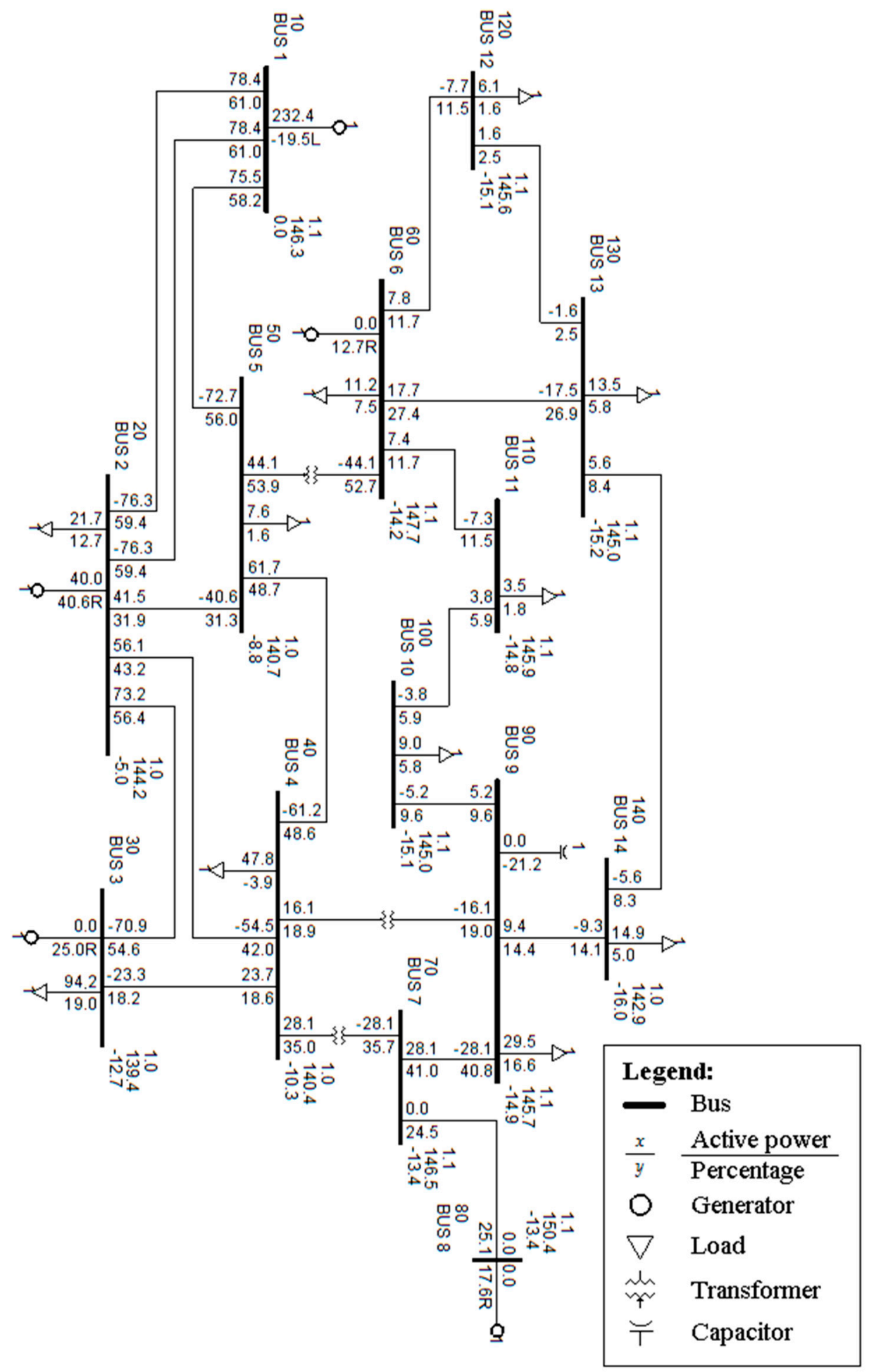

Figure 9. Power flow calculation of the base scenario.

\subsection{N-1 Analysis}

$\mathrm{N}-1$ security assessments indicated two main problematic cases in which safety operation criteria were not met. Overload of one line between buses 1 and 2 happened when the other line between the same buses was out of operation. Figure 10 shows N-1 analysis of line 1-2 (1) results, after which line 1-2 (2) overloaded (106.7\%). In the next step it was necessary to find out whether the specified overload could be mitigated by using the proposed bus-splitting protection scheme. 


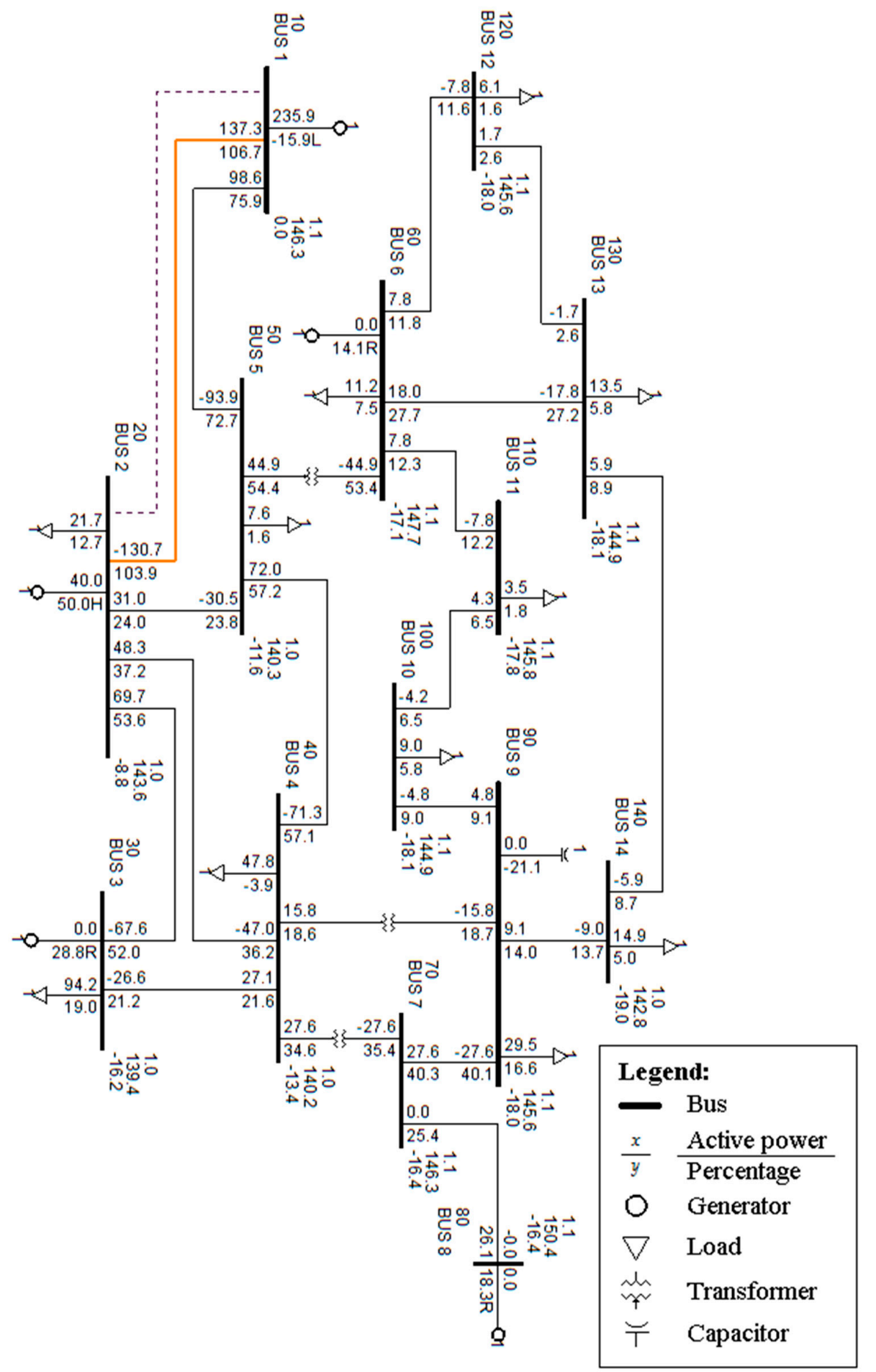

Figure 10. N-1 power flow analysis.

\subsection{Power Flow Model and Adjustment Factor Calculation}

Adjustment factor $k_{P e}$ needed to be calculated in order to adjust the optimization algorithm to the real overloaded state of the network. Use of actual synchronized measurements during the overload state is recommended. Every PMU measurement has its own time tag, which makes such measurements easy to compare. As the calculations were run on the test system, synchronized measurement data were replaced by data from nonlinear AC power flow model calculations. DC power flow model data were calculated as part of the optimization algorithm. The calculation results of the IEEE 14 bus test model for the N-1 overload case are shown in Table 3. 
Table 3. Calculation of adjustment factor $k_{P e}$ during the overload state.

\begin{tabular}{|c|c|c|c|c|c|}
\hline Element & From Bus & To Bus & $P_{\text {REAL }}(\mathrm{MW})$ & $P_{D C}(\mathrm{MW})$ & $k_{P e}$ \\
\hline 1 & 1 & 2 & 0 & 0 & 1 \\
\hline 2 & 1 & 2 & 137.3 & 127.3 & 0.91 \\
\hline 3 & 1 & 5 & 98.6 & 91.7 & 0.94 \\
\hline 4 & 2 & 3 & 69.7 & 66.6 & 0.97 \\
\hline 5 & 2 & 4 & 48.3 & 48 & 1 \\
\hline 6 & 2 & 5 & 31 & 31.1 & 1 \\
\hline 7 & 3 & 4 & -26.6 & -27.6 & 0.99 \\
\hline 8 & 4 & 5 & -71.3 & -72.5 & 0.99 \\
\hline 9 & 4 & 7 & 27.6 & 28.6 & 0.99 \\
\hline 10 & 4 & 9 & 15.8 & 16.4 & 0.99 \\
\hline 11 & 5 & 6 & 44.9 & 42.7 & 0.97 \\
\hline 12 & 6 & 11 & 7.8 & 6.7 & 0.98 \\
\hline 13 & 6 & 12 & 7.8 & 7.6 & 1 \\
\hline 14 & 6 & 13 & 18 & 17.2 & 0.99 \\
\hline 15 & 7 & 8 & 0 & 0 & 1 \\
\hline 16 & 7 & 9 & 27.6 & 28.6 & 0.98 \\
\hline 17 & 9 & 10 & 4.8 & 5.9 & 0.98 \\
\hline 18 & 9 & 14 & 9.1 & 9.7 & 0.99 \\
\hline 19 & 10 & 11 & -4.2 & -3.2 & 0.98 \\
\hline 20 & 12 & 13 & 1.7 & 1.5 & 1 \\
\hline 21 & 13 & 14 & 5.9 & 5.2 & 0.99 \\
\hline
\end{tabular}

\subsection{Optimal Bus-Splitting Scheme Solution-SIPS IEEE 14 B}

Taking into account the input data on the amount of production and load at each node, reactance of lines and transformers, together with the network topology state and optimization algorithm as a solution, determines which bus needs to be separated and in which arrangement. For the IEEE 14 bus test system, according to the described mathematical problem in Section 4, the optimization algorithm with the objective function of minimizing the total production decided that it was necessary to separate bus 2 into two separate systems and, thus, remove the overload of line 1-2 (2). The exact optimal solution for the bus-splitting protection scheme is shown in Table 4.

Table 4. Optimal bus-splitting scheme solution-SIPS IEEE 14 B.

\begin{tabular}{cc}
\hline SIPS & IEEE 14 B \\
\hline Bus & 2 \\
\hline Bus 21 & Line 1-2 (2), 2-3 i 2-4 and generator \\
Bus 22 & Line 2-5 and load \\
\hline
\end{tabular}

The execution time of the optimization algorithm for the IEEE 14 bus test system was $0.3 \mathrm{~s}$, and the optimum solution was achieved in 208 iterations. Tests were performed on a PC with $2.4 \mathrm{GHz}$ CPU. That the optimal bus splitting solution was found relatively fast provides the possibility of applying the developed algorithm within the protection schemes supported by PMU technology. The described solution of the optimization algorithm was defined by SIPS IEEE 14 B (Figure 11). 


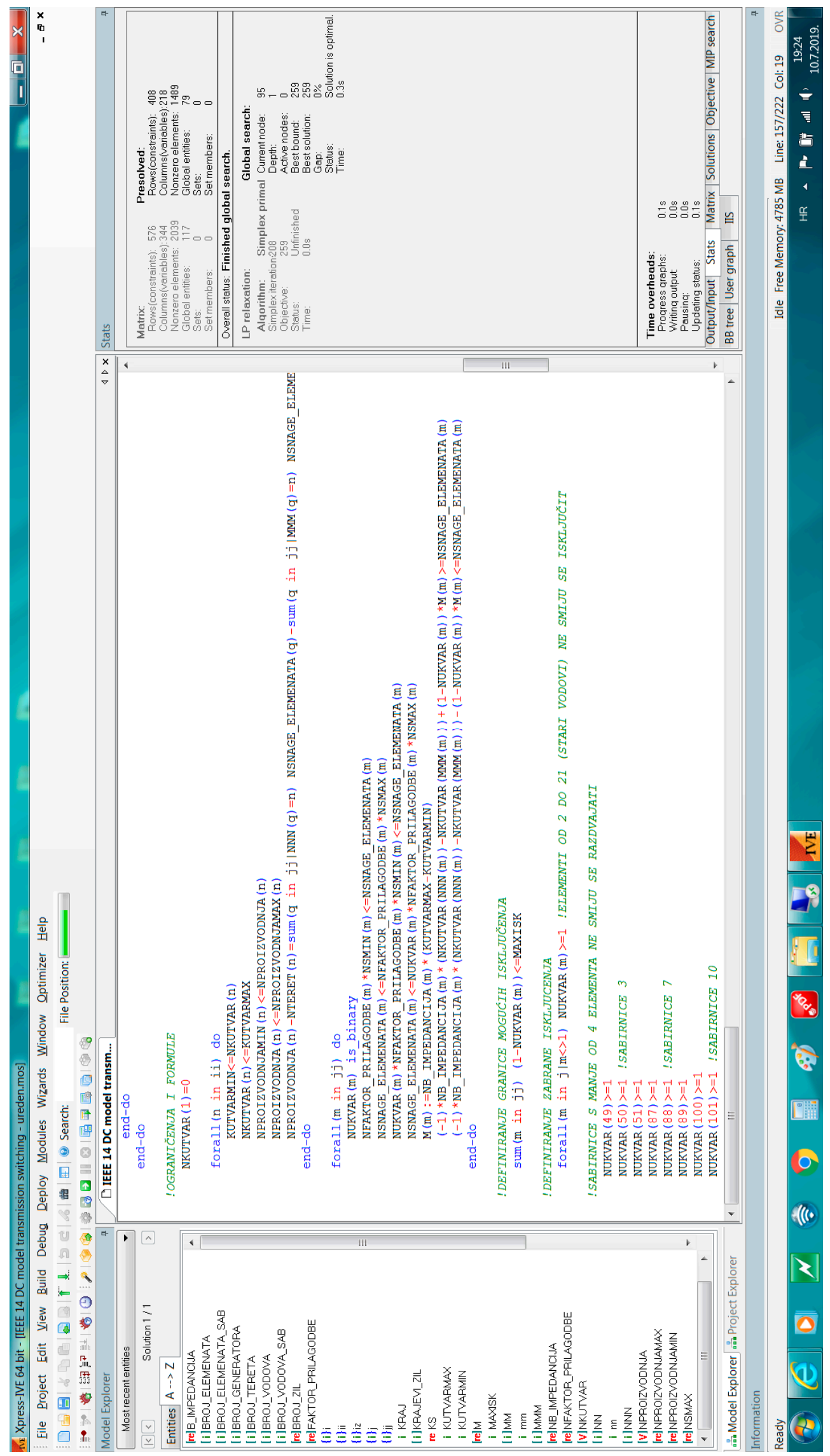

Figure 11. Optimal bus-splitting scheme solution-SIPS IEEE 14 B. 
The SIPS IEEE 14 B solution was checked by nonlinear AC power flow calculations (Figure 12). Separation of bus 2 into two separate systems was simulated according to the defined bus-splitting scheme solution in Table 4. AC power flow calculations with separated systems at bus 2 gave $121.7 \mathrm{MW}$ of active power and $95 \%$ of apparent power on line 1-2 (2). These given results confirm the overload was mitigated, and the bus-splitting action of the described algorithm was correct.

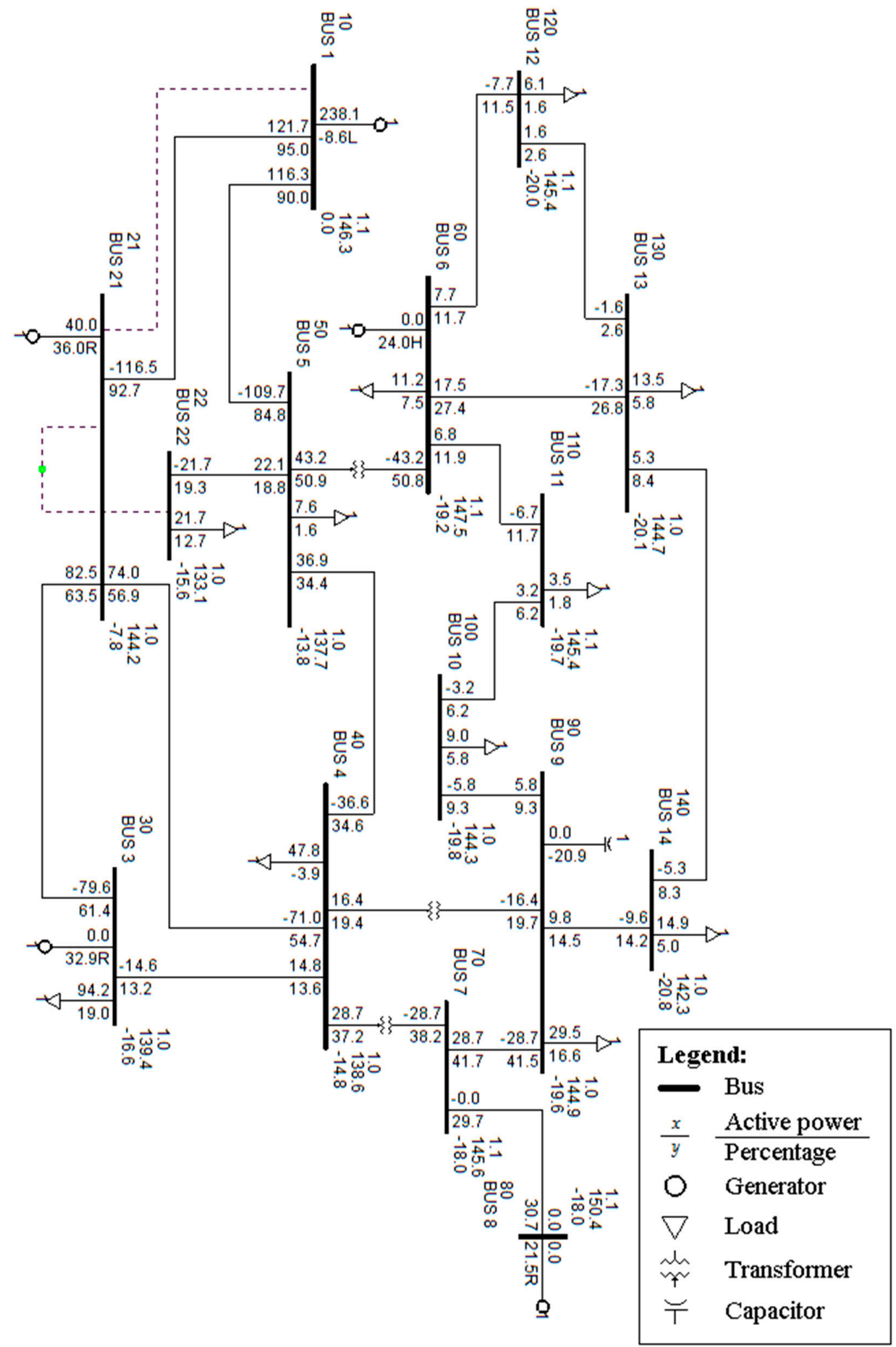

Figure 12. SIPS IEEE $14 \mathrm{~B}$ solution check using a nonlinear AC power flow model. 


\subsection{IEEE 14 Bus Test System Security Assessment}

The IEEE 14 bus test system security assessment with and without SIPS implementation consisted of comparisons of voltage conditions and power flows together with production, consumption, and loss levels. Figure 13 shows voltage level comparisons at buses 1 to 14 . A significant reduction in the voltage level of new bus 22 was evident in the given picture. Despite the significant decrease in the voltage level of bus 22, use of the calculated optimal bus-splitting model did not endanger the security of the analysed test system in its entirety.

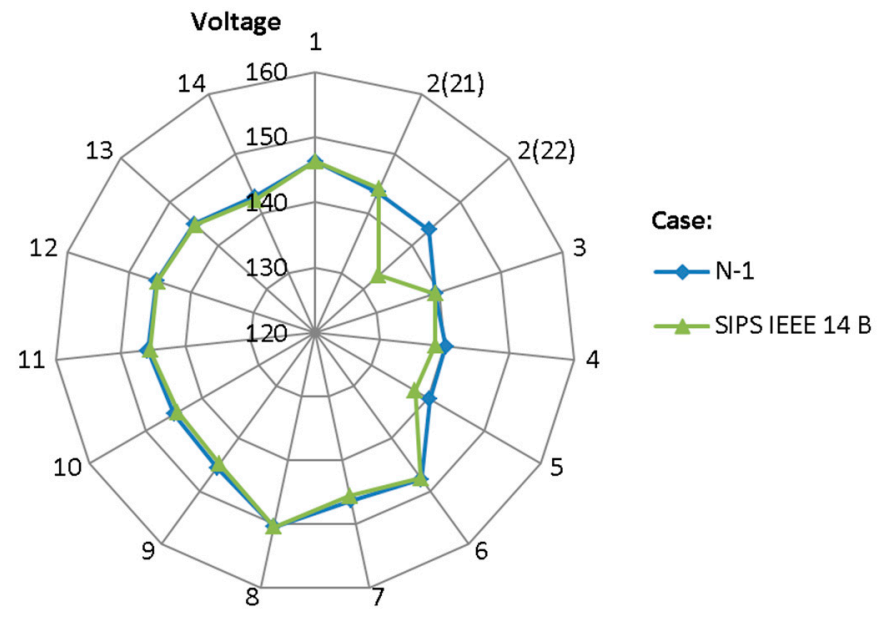

Figure 13. Voltage level comparison.

Figure 14 shows relations between voltage angles at buses 1 to 14 . It is evident that usage of the proposed SIPS changed the voltage conditions compared to the $\mathrm{N}-1$ analysis. The voltage angles between buses generally increased, but a noticed remark did not cause additional problems for the system's security. Voltage deviations are a common occurrence in EPS operations. The operational voltage ranges in transmission and sub-transmission power networks are usually from 90 to $110 \%$ of the nominal value. Voltage levels are direct indicators of reactive power flows in a network, while voltage angles are indicators of active power flows.

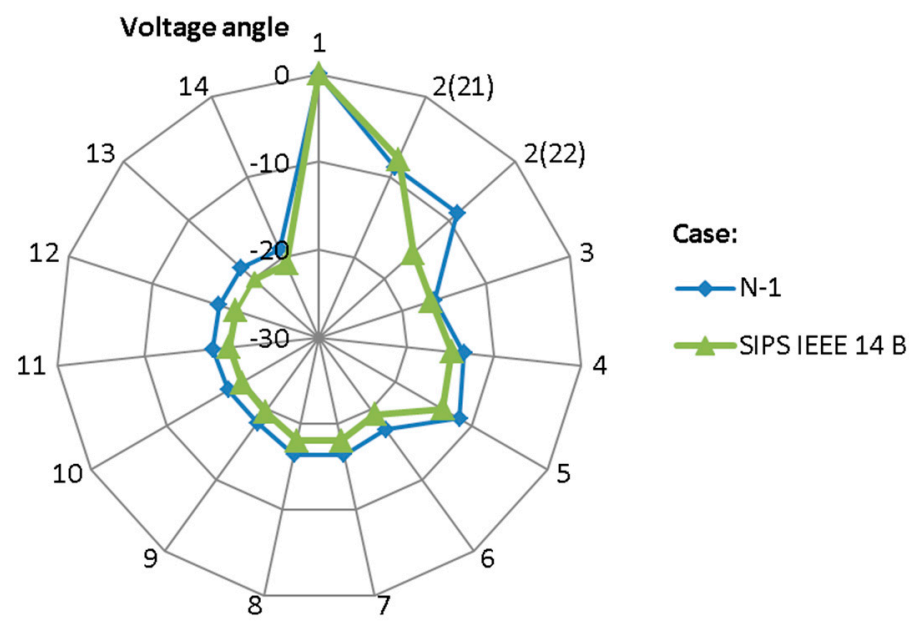

Figure 14. Voltage angle comparison.

Figure 15 shows, in red color, the assumed power flow limits of all lines and transformers in the test system. N-1 analysis of line 1-2 (1) clearly showed overloading of line 1-2 (2). Without SIPS implementation, tripping of the overloaded line from the local relay protection system would lead 
to a voltage breakdown of the observed network part. The same figure shows distribution of the power flows in the case of using the proposed SIPS IEEE $14 \mathrm{~B}$. It is evident that using the proposed bus-splitting scheme returned the power flow of line 1-2 (2) within the allowed limits.

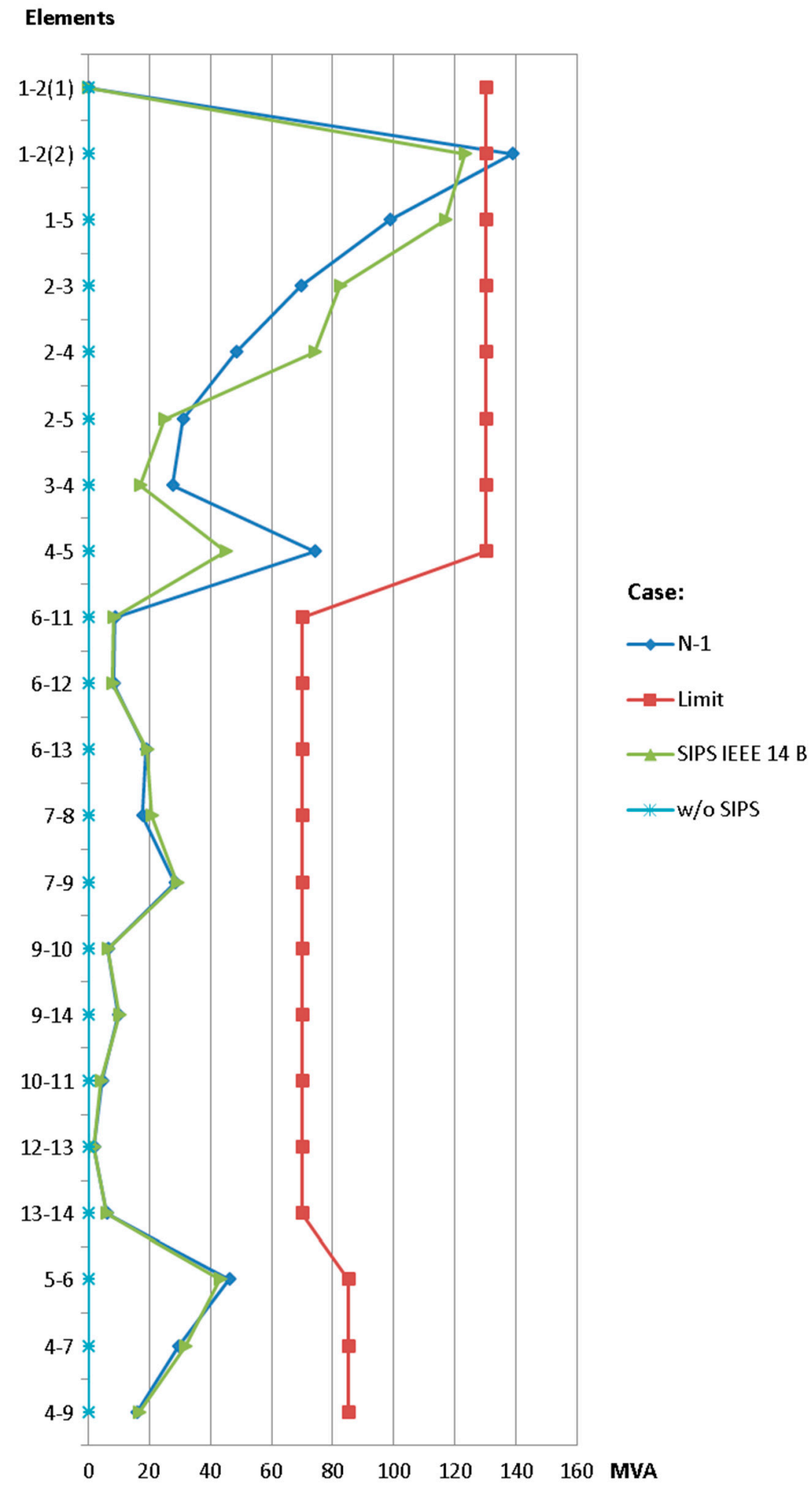

Figure 15. Power flow comparison. 
The existing level of production and consumption of the observed test system was maintained by applying SIPS IEEE 14 B. Network losses were slightly increased, as shown in Figure 16. The use of the calculated optimal bus-splitting model resulted in satisfactory security criteria for power flows and voltage conditions, and it is recommended for use in overload cases of any of the lines between buses 1 and 2 of the tested system.

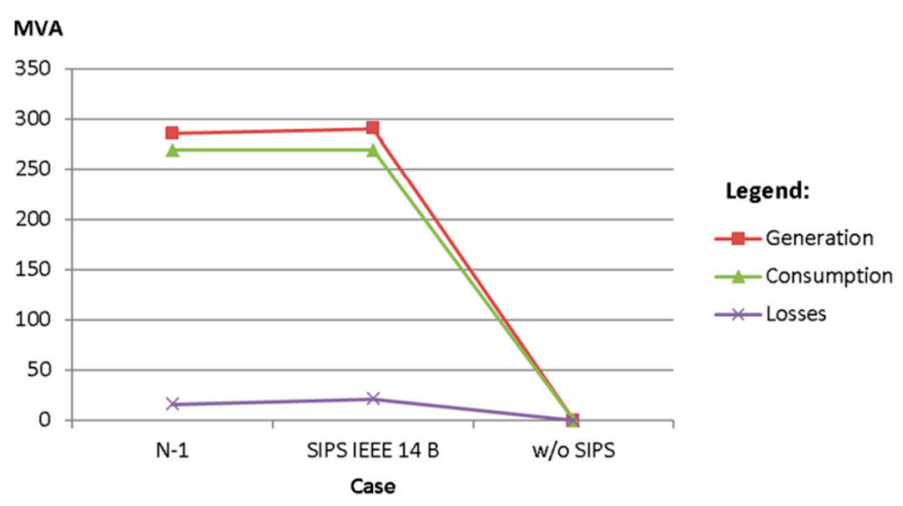

Figure 16. Production, consumption, and loss comparisons.

\section{Conclusions}

This paper discusses methodology of SIPS development that incorporates application of PMU technology. In the developed model, different scenarios were simulated to investigate the transmission system under different conditions and levels of overloading and congestion. Using the proposed methodology, SIPS based on optimal bus splitting schemes was successfully tested and verified on an IEEE 14 bus test system. Simulation results and performed analyses showed that congestion and overload problems of the transmission network together with coordination of local relay protection systems can be solved using the proposed SIPS supported by PMU measurements. The model presented in the paper could have a key role in creating new SIPS as a part of the development process of future smart transmission grid WAMPAC systems. Future research will be directed towards the development of various SIPS schemes supported by PMU technology such as generation of reduction schemes and load-shedding schemes, including under-frequency and under-voltage protection schemes.

Author Contributions: Z.Z. and I.K. conceived and designed the experiments; Z.Z. performed the experiments; Z.Z. and I.K. analyzed the data; Z.Z. prepared the original paper draft; Z.Z. and I.K. reviewed and edited.

Acknowledgments: This work was supported in part by the Croatian Science Foundation under the project WINDLIPS-WIND energy integration in Low Inertia Power System (grant No. HRZZ-PAR-02-2017-03). The work of the authors is a part of the H2020 project CROSSBOW-CROSS BOrder management of variable renewable energies and storage units enabling a transnational Wholesale market (Grant No. 773430). This document has been produced with the financial assistance of the European Union. The contents of this document are the sole responsibility of authors and can under no circumstances be regarded as reflecting the position of the European Union.

Conflicts of Interest: The authors declare no conflicts of interest.

\section{References}

1. He, P.; Wen, F.; Ledwich, G.; Xue, Y. Small signal stability analysis of power systems with high penetration of wind power. J. Mod. Power Syst. Clean Energy 2013, 1, 241-248. [CrossRef]

2. Yuan, X. Overview of problems in large-scale wind integrations. J. Mod. Power Syst. Clean Energy 2013, 1, 22-25. [CrossRef]

3. Martin, K.E.; Hamai, D.; Adamiak, M.G.; Anderson, S.; Begovic, M.; Benmouyal, G.; Brunello, G.; Burger, J.; Cai, J.Y.; Dickerson, B.; et al. Exploring the IEEE standard C37.118-2005 synchrophasors for power systems. IEEE Trans. Power Deliv. 2008, 23, 1805-1811. [CrossRef] 
4. Phadke, A.G.; Thorp, J.S. Synchronized Phasor Measurements and Their Applications; Springer: New York, NY, USA, 2008; pp. 3-27.

5. Terzija, V.; Valverde, G.; Cai, D.; Regulski, P.; Madani, V.; Fitch, J.; Skok, S.; Begovic, M.M.; Phadke, A. Wide-area monitoring, protection, and control of future electric power networks. Proc. IEEE 2011, 99, 80-93. [CrossRef]

6. Ivankovic, I.; Kuzle, I.; Holjevac, N. Multifunctional WAMPAC system concept for out-of-step protection based on synchrophasor measurements. Int. J. Electr. Power Energy Syst. 2017, 87, 77-88. [CrossRef]

7. Wang, L.; Bo, Z.Q.; Ma, X.W.; Wang, Q.P.; Zhao, Y.K.; Zhou, F.Q.; Feng, X. A New Integrated Protection Scheme for Power Transmission Lines. In Proceedings of the 2017 IEEE PES General Meeting, Chicago, IL, USA, 16-20 July 2017.

8. Quiros-Tortos, J.; Wall, P.; Terzija, V. Reducing excessive standing phase angle differences: A new approach based on OPF and wide area measurements. Int. J. Electr. Power Energy Syst. 2016, 78, 13-21.

9. Wang, W.; Liu, M.; Zhao, X.; Yang, G. Shared-network scheme of SMV and GOOSE in smart substation. J. Mod. Power Syst. Clean Energy 2014, 2, 438-443. [CrossRef]

10. Zbunjak, Z.; Kuzle, I. Possible savings in electricity transmission using wide area monitoring technologies in Croatian power transmission network. In Proceedings of the 8th International Conference on the European Energy Market, Zagreb, Croatia, 25-27 May 2011.

11. Klarić, M.; Kuzle, I.; Holjevac, N. Wind Power Monitoring and Control Based on Synchrophasor Measurement Data Mining. Energies 2018, 11, 3525.

12. Ivanković, I.; Kuzle, I.; Holjevac, N. Wide Area Information-Based Transmission System Centralized Out-of-Step Protection Scheme. Energies 2017, 10, 633. [CrossRef]

13. Zbunjak, Z.; Kuzle, I. Advanced Control and System Integrity Protection Schemes of Croatian Power Transmission Network with Integrated Renewable Energy Sources. In Proceedings of the Eurocon 2013, Zagreb, Croatia, 1-4 July 2013.

14. Shahriar, M.S.; Habiballah, I.O.; Hussein, H. Optimization of Phasor Measurement Unit (PMU) Placement in Supervisory Control and Data Acquisition (SCADA)-Based Power System for Better State-Estimation Performance. Energies 2018, 11, 570. [CrossRef]

15. Ali, Z.M.; Razavi, S.E.; Javadi, M.S.; Gandoman, F.H.; Aleem, S.H.E.A. Dual Enhancement of Power System Monitoring: Improved Probabilistic Multi-Stage PMU Placement with an Increased Search Space \& Mathematical Linear Expansion to Consider Zero-Injection Bus. Energies 2018, 11, 1429.

16. Nuqui, R.F.; Phadke, A.G. Phasor measurement unit placement techniques for complete and incomplete observability. IEEE Trans. Power Deliv. 2005, 20, 2381-2388. [CrossRef]

17. Pal, A.; Sanchez-Ayala, G.A.; Centeno, V.A.; Thorp, J.S. A PMU placement scheme ensuring real-time monitoring of critical buses of the network. IEEE Trans. Power Deliv. 2014, 29, 510-517. [CrossRef]

18. Anderson, P.M.; LeReverend, B.K. Industry experience with special protection schemes. IEEE Trans. Power Syst. 1996, 11, 1166-1179. [CrossRef]

19. Madani, V.; Novosel, D.; Horowitz, S.; Adamiak, M.; Amantegui, J.; Karlsson, D.; Imai, S.; Apostolov, A. IEEE PSRC report on global industry experiences with system integrity protection schemes (SIPS). IEEE Trans. Power Deliv. 2010, 25, 2143-2155. [CrossRef]

20. Koglin, H.-J.; Muller, H. Overload Reduction through Corrective Switching Actions. In Proceedings of the IEE International Conference on Power System Monitoring and Control, London, UK, 24-26 June 1980.

21. Van Amerongen, R.A.M.; Van Meeteren, H.P. Security Control by Real Power Rescheduling, Network Switching and Load Shedding. In Proceedings of the International Conference on Large High Voltage Electric Systems, Paris, France, 27 August-4 September 1980. Report 32-02.

22. Rolim, J.G.; Machado, L.J.B. A study of the use of corrective switching in transmission systems. IEEE Trans. Power Syst. 1999, 14, 336-341. [CrossRef]

23. Wei, S.; Vittal, V. Corrective switching algorithm for relieving overloads and voltage violations. IEEE Trans. Power Syst. 2005, 20, 1877-1885.

24. Wrubel, J.N.; Rapcienski, P.S.; Lee, K.L.; Gisin, B.S.; Woodzell, G.W. Practical experience with corrective switching algorithm for on-line applications. IEEE Trans. Power Syst. 1996, 11, 415-421. [CrossRef]

25. MathWorks@. MATLAB 2016 Software. Available online: https://uk.mathworks.com/products/matlab.html (accessed on 12 February 2018). 
26. Purchala, K. Modeling and Analysis of Techno-Economic Interactions in Meshed High Voltage Grids Exhibiting Congestion. Ph.D. Thesis, University of Leuven, Leuven, Belgium, 2005.

27. Power System Test Case Archive, University Washington. Available online: http://www.ee.washington.edu/ research/pstca/pf14/pg_tca14bus.htm (accessed on 23 September 2014).

28. PSS®E-High-Performance Transmission Planning and Analysis Software. Available online: https://new.siemens.com/global/en/products/energy/services/transmission-distribution-smart-grid/ consulting-and-planning/pss-software/pss-e.html (accessed on 3 September 2019).

(C) 2019 by the authors. Licensee MDPI, Basel, Switzerland. This article is an open access article distributed under the terms and conditions of the Creative Commons Attribution (CC BY) license (http://creativecommons.org/licenses/by/4.0/). 